\title{
EFEITO DA INTENSIDADE DE DESBASTE NAS CARACTERÍSTICAS DENDROMÉTRICAS E TECNOLÓGICAS DA MADEIRA DE Eucalyptus grandis
}

\author{
EFFECT OF THE THINNING INTENSITY IN THE DENDROMETRIC AND TECHNOLOGICAL \\ CHARACTERISTICS OF THE WOOD OF Eucalyptus grandis
}

\author{
Rômulo Trevisan ${ }^{1}$ Clovis Roberto Haselein ${ }^{2}$ Elio José Santini ${ }^{2}$ \\ Paulo Renato Schneider ${ }^{3}$ Leonel Freitas de Menezes ${ }^{4}$
}

\section{RESUMO}

Este estudo teve como objetivo avaliar o efeito de diferentes intensidades de desbaste nas características dendrométricas e tecnológicas da madeira de Eucalyptus grandis W. Hill ex Maiden, aos 14 anos de idade, situado próximo ao litoral do Rio Grande do Sul. O delineamento utilizado foi em blocos ao acaso, com quatro tratamentos (T1 = 1638 árvores/ha; T2 = 1150 árvores/ha; T4 = 589 árvores/ha e T8 = 192 árvores/ha) e quatro repetições. As árvores foram selecionadas com base nos diâmetros dominante e médio de cada tratamento, e, após o abate, foram medidas e cubadas pelo método de Smalian, sendo retirados os discos nas posições $0,25,50,75$ e $100 \%$ da altura comercial e no DAP, destinados à determinação da massa específica básica, massa seca e retratibilidade radial. Com relação às características dendrométricas, os resultados mostraram que a aplicação de desbastes mais pesados ocasionaram ganhos significativos em diâmetro à altura do peito, altura e volume para as árvores médias, não tendo influenciado as árvores dominantes. Em relação às características tecnológicas, verifica-se que a massa específica básica das árvores médias foi influenciada pelo desbaste, porém sem apresentar tendência clara em relação à intensidade da intervenção. A produção de massa seca das árvores médias aumentou com a intensidade do desbaste e não afetou a produção das árvores dominantes. A retratibilidade radial das árvores dominantes e médias cresceu no sentido medula-casca, não sendo alterada pelos desbastes. Os resultados permitem concluir que as intervenções silviculturais aplicadas no povoamento florestal influem na qualidade da madeira.

Palavras-chave: Eucalyptus grandis; desbaste; qualidade da madeira.

\section{ABSTRACT}

This study aimed at evaluating the effect of different thinning intensities in the dendrometric and technological characteristics of 14 years-old Eucalyptus grandis W. Hill ex Maiden wood sited in the northern coast of Rio Grande do Sul. Randomized blocks experiment, with four treatments $(\mathrm{T} 1=1638$ trees/ha; T2 $=1150$ trees/ha; T4 $=589$ trees/ha and T $8=192$ trees/ha) and four repetitions was analyzed. The trees were selected on the basis of the dominant and average diameter of each treatment. After cutting down, trees were measured and the volume was determined by the Smalian method, disks were taken at the base 0, $25,50,75$ and $100 \%$ of the commercial height, and at the DBH (diameter at breast height), for basic density, dry mass and radial shrinkage determination. In relation to the dendrometric characteristics, results indicate that the application of more severe thinning caused significant gain in diameter at breast height, height and volume in average trees, not influencing the dominant ones. Concerning the technological characteristics, results indicate that the basic density of the average trees was influenced by thinning; however, without presenting clear trend regarding the intensity of the intervention. The production of dry mass of the average trees increased with the thinning intensity and did not affect the production of the dominant trees. The radial shrinkage of the dominant and average trees grew up in the pith-to-bark direction, not being modified by thinning intensity. Results allow concluding that the silvicultural interventions applied in the forest influence wood quality.

Keywords: Eucalyptus grandis; thinning; wood quality.

1. Engenheiro Florestal, MSc., Professor do Curso de Engenharia Florestal da Universidade do Oeste de Santa Catarina, CEP 89.820-000, Xanxerê (SC). romulo_trevisan@yahoo.com.br

2. Engenheiro Florestal, Dr., Professor Associado do Departamento de Ciências Florestais, Centro de Ciências Rurais, Universidade Federal de Santa Maria, CEP 97.105-900, Santa Maria (RS). haseleic@ccr.ufsm.br

3. Engenheiro Florestal, Dr., Professor Titular do Departamento de Ciências Florestais, Centro de Ciências Rurais, Universidade Federal de Santa Maria, CEP 97.105-900, Santa Maria (RS).paulors@ccr.ufsm.br

4. Engenheiro Florestal, International Business in Eucalyptus (IBE), Av. Dr. Salvador França, 889/401, CEP 90690000, Porto Alegre (RS).fmleonel@terra.com.br

Recebido para publicação em 8/03/2007 e aceito em 1/11/2007. 


\section{INTRODUÇÃO}

O gênero Eucalyptus é atualmente uma das principais essências florestais encontradas no Brasil. Entre as folhosas, poderá ser a matéria-prima com maior demanda no mercado, sendo utilizada para atender diversos segmentos industriais e comerciais.

A qualidade e a produtividade dos povoamentos florestais, segundo Soares et al. (2003), são fatores determinantes para a conquista de bons mercados e para se obter um fluxo contínuo de matéria-prima. Para que isto seja alcançado, é necessário executar tratos silviculturais, dentre os quais o desbaste é um dos mais importantes por distribuir o potencial de crescimento do sítio florestal em um número menor de árvores (FISHWICK, 1976; MONTEIRO et al., 1984; BLANCO et al., 1988; SCHEEREN, 2003), por proporcionar espaço para o desenvolvimento da copa e do sistema radicular e, conseqüentemente, por melhorar o aproveitamento de luz, nutrientes e água disponíveis no sítio (SMITH, 1962; SCHNEIDER et al., 1998; SCHNEIDER, 2002).

Segundo Panshin e De Zeeuw (1980), Ferreira (1996) e Haselein et al. (2002), existem diversas características na madeira, porém a massa específica é o principal parâmetro usado para expressar a qualidade por ter correlação com outras propriedades da madeira e também por ser de fácil determinação.

Conforme Kollmann e Côté (1968), a massa específica da madeira pode ser alterada em função das características da própria espécie, bem como das influências externas, assim como das variações ambientais e das intervenções silviculturais (WILKINS e KITAHARA, 1991; MALAN e HOON, 1992; DOWNES e RAYMOND, 1997; DEBELL et al., 2001).

A retratibilidade da madeira é o fenômeno relacionado à sua variação dimensional, em função da troca de umidade do material com o meio que o envolve (OLIVEIRA, 1998; SILVA e OLIVEIRA, 2003). Todo material higroscópico, como a madeira e vários outros materiais celulósicos, apresenta contração quando o seu teor de umidade é reduzido do ponto de saturação das fibras (PSF) à condição absolutamente seca ou anidra, resultando em um dos mais importantes problemas práticos que ocorrem durante a sua utilização (OLIVEIRA e SILVA, 2003).

Desta maneira, este trabalho foi desenvolvido com árvores do estrato dominante e médio tendo como objetivo avaliar o efeito do desbaste nas características dendrométricas e tecnológicas da madeira de Eucalyptus grandis W. Hill ex Maiden.

\section{MATERIAL E MÉTODO}

\section{Características do experimento}

O material utilizado neste estudo é proveniente de um povoamento experimental pertencente à Empresa Flosul Indústria e Comércio de Madeiras Ltda., localizada no município de Capivari do Sul, litoral norte do Estado do Rio Grande do Sul. O experimento situa-se, aproximadamente, nas coordenadas geográficas de $50^{\circ} 30^{\prime}$ de longitude oeste e $30^{\circ} 08^{\prime}$ de latitude sul.

O povoamento experimental foi implantado em maio de 1990, com espaçamento inicial de 3,0 x 1,7 m. O delineamento estatístico utilizado para a realização deste estudo foi em blocos ao acaso, com quatro repetições para cada tratamento.

Os desbastes foram executados de acordo com o plano de redução de área basal, favorecendo o desenvolvimento das árvores de maiores dimensões. A Tabela 1 apresenta os tratamentos analisados e as condições atuais do experimento.

A numeração dos tratamentos (Tabela 1) foi descrita conforme a denominação da empresa e está relacionada com as intensidades de desbaste estudadas: 1 - testemunha sem desbaste; 2 , 4 e 8 - manutenção de, aproximadamente, $70 \% ; 40 \%$ e $10 \%$ da área basal da testemunha, respectivamente. 
TABELA 1: Tratamentos de desbaste analisados aos 14 anos de idade.

TABLE 1: Thinning treatments analyzed at 14 years-old.

\begin{tabular}{c|c|c|c|c|c|c}
\hline \multirow{2}{*}{ Tratamentos } & Desbastes & \multicolumn{5}{|c}{ Densidade (árvores/ha) } \\
\cline { 3 - 7 } & executados & Bloco 1 & Bloco 2 & Bloco 3 & Bloco 4 & Média \\
\hline 1 & 0 & 1.925 & 1.675 & 1.400 & 1.550 & 1.638 \\
2 & 1 & 1.125 & 1.150 & 1.175 & 1.150 & 1.150 \\
4 & 3 & 589 & 589 & 589 & 589 & 589 \\
8 & 6 & 190 & 185 & 196 & 196 & 192 \\
\hline
\end{tabular}

A amostragem das árvores foi realizada no décimo quarto ano de idade, com base no diâmetro à altura do peito (DAP). Foram retiradas de cada tratamento uma árvore com diâmetro dominante e uma árvore com diâmetro médio por bloco, totalizando 32 árvores.

\section{Determinação das características dendrométricas}

Para o estudo dos parâmetros dendrométricos, foram tomados os diâmetros e as alturas das árvores selecionadas. Após a derrubada de cada árvore, a mensuração dos diâmetros foi realizada com o auxílio de uma suta graduada em centímetros, e a altura, medida com auxílio de uma fita métrica.

Com os dados de diâmetro e altura, as árvores foram cubadas pelo método de Smalian (FINGER, 1992), sendo determinados o volume total com casca (v) e o volume total sem casca (v sc). O volume comercial $\left(\mathrm{v}_{13}\right)$ com casca e sem casca até $13 \mathrm{~cm}$ (que corresponde ao diâmetro mínimo de entrada de toras na serraria da empresa) foi obtido limitando-se o diâmetro a esta medida. Para a mensuração da espessura da casca, foi utilizado um paquímetro digital.

\section{Determinação das características tecnológicas}

\section{Massa específica básica}

Para o estudo desta característica tecnológica, foi retirado um disco com, aproximadamente, dois centímetros de espessura, nas posições da base (0), 25, 50, 75 e 100\% da altura comercial (definida pelo diâmetro mínimo do tronco de $13 \mathrm{~cm}$ ) e no DAP.

Estes discos foram embalados em sacos plásticos e transportados para o Laboratório de Produtos Florestais (LPF) da Universidade Federal de Santa Maria (UFSM), onde foram marcadas e seccionadas duas cunhas simetricamente opostas, contendo partes de cerne e alburno.

A massa específica básica de cada cunha foi determinada obtendo-se o volume verde pelo Método da Balança Hidrostática e o peso seco em estufa a $103{ }^{\circ} \mathrm{C}$ (VITAL, 1984).

Após a obtenção da massa específica básica em cada posição do tronco pela média dos valores das duas cunhas, foi realizada a determinação da massa específica básica ponderada em função do volume de cada árvore, conforme Vital (1984).

\section{Massa seca}

Esta característica foi analisada tanto para árvores dominantes como para árvores médias, sendo obtida pela multiplicação entre a massa específica básica ponderada e o volume comercial sem casca das árvores.

$$
\mathrm{MS}=\rho_{\mathrm{bPond}} \cdot \mathrm{v}_{13} \mathrm{sc}
$$

Em que: $\mathrm{MS}$ = massa seca da árvore, em $\mathrm{kg}$; $\rho_{\mathrm{bPond}}=$ massa específica básica ponderada da árvore, em $\mathrm{kg} / \mathrm{m}^{3}$; $\mathrm{v}_{13} \mathrm{sc}=$ volume comercial sem casca da árvore, $\mathrm{em} \mathrm{m}^{3}$.

\section{Retratibilidade}

O estudo da retratibilidade radial, no sentido medula-casca, foi realizado somente na posição do DAP. Esses discos foram transportados para o LPF da Universidade Federal de Santa Maria (UFSM), onde foram marcados e seccionados em baguetas (contendo a medula) de $3 \mathrm{~cm}$ de espessura.

Após a confecção das baguetas, foram realizadas a marcação e a numeração dos corpos-de-prova, com dimensões de 3,0 x 3,0 x 2,0 cm (radial, tangencial e longitudinal, respectivamente), no sentido medula- 
casca. Procedeu-se a mensuração das distâncias da medula até a casca para o cálculo das posições relativas. Em seguida, os corpos-de-prova foram seccionados e submersos em água, onde permaneceram até atingir peso constante.

Para o teste de retratibilidade radial, cada corpo-de-prova, inicialmente em estado verde, foi pesado em balança eletrônica digital, com precisão de $0,01 \mathrm{~g}$, e mensurado na respectiva direção radial. Esta mensuração foi realizada com auxílio de um relógio comparador com precisão de $0,01 \mathrm{~mm}$ em dois pontos previamente marcados nos corpos-de-prova.

Realizadas estas aferições, os corpos-de-prova foram então acondicionados em câmara climatizada a uma temperatura de $20{ }^{\circ} \mathrm{C}$ e umidade relativa de $65 \%$, até atingir a umidade de equilíbrio do ambiente (12\%).

A partir desta umidade, a secagem foi realizada em estufa com temperatura de $103{ }^{\circ} \mathrm{C}$ para tomadas da massa e da dimensão radial na condição $0 \%$ de umidade.

$$
\mathrm{Ct}_{\mathrm{Rd}}=\frac{\mathrm{Du}-\mathrm{Do}}{\mathrm{Du}} 100
$$

Em que: $\mathrm{Ct}_{\mathrm{Rd}}=$ contração radial total, em \%; Du = dimensão na condição saturada (acima PSF), em cm; Do = dimensão na condição de $0 \%$ de umidade, em cm.

\section{Análise estatística dos dados}

\section{Análise de regressão e variância dos parâmetros dendrométricos}

Considerando a possibilidade de se utilizar o espaçamento (espaço vital) como variável contínua, os dados de volume total com casca das árvores-amostra, em cada tratamento de desbaste, foram estimados por regressão. Para tanto, os dados de volume total com casca por árvore foram processados no pacote "Statistical Analysis System" (SAS, 1993), pelo procedimento de regressão com variável Dummy, com base no modelo de Spurr, expresso por:

$$
\mathrm{v}=\mathrm{b}_{0}+\mathrm{b}_{1}\left(\mathrm{~d}^{2} \mathrm{~h}\right)
$$

Neste modelo de Spurr, foram adicionadas variáveis Dummy, definidas pelos tratamentos de desbaste aplicados, tendo sido expresso pelas seguintes variáveis independentes:

$$
v=f\left(d^{2} h, D, D \cdot d^{2} h\right)
$$

Em que: $\mathrm{v}=$ volume total com casca por árvore, $\mathrm{em}^{3} ; \mathrm{d}=$ diâmetro à altura do peito (DAP) das árvoresamostra, em $\mathrm{m} ; \mathrm{h}=$ altura total das árvores-amostra, em m; D = tratamentos (dummy); $\mathrm{D} . \mathrm{d}^{2} \mathrm{~h}=$ interação tratamento (dummy) com a variável $\mathrm{d}^{2} \mathrm{~h}$.

As demais características dendrométricas mensuradas das árvores dominantes e médias, na idade de 14 anos, foram submetidas à análise de variância para delineamento experimental em blocos ao acaso e, no caso de rejeição da hipótese de igualdade de médias entre tratamentos, ao teste de médias (Least Significant Difference - LSD, $\alpha=5 \%$ ).

\section{Análise de regressão e variância para os parâmetros tecnológicos}

Para o estudo do comportamento da retratibilidade radial no sentido medula-casca em função do espaço vital, os dados de contração das árvores dominantes e médias foram submetidos à análise de regressão.

Estes dados foram processados pelo pacote "Statistical Analysis System" (SAS, 1993), no qual foi aplicado o procedimento Stepwise de modelagem de regressão. O modelo máximo de regressão foi definido pela equação a seguir.

$$
\mathrm{Ct}_{\mathrm{Rd}}, \log \left(\mathrm{Ct}_{\mathrm{Rd}}\right)=\mathrm{f}\left(\mathrm{P}, 1 / \mathrm{P}, \mathrm{P}^{2}, 1 / \mathrm{P}^{2}, \log (\mathrm{P}), 1 / \log (\mathrm{P})\right)
$$

Em que: $\mathrm{Ct}_{\mathrm{Rd}}=$ retratibilidade radial das árvores médias e dominantes, em $\% ; \mathrm{P}=$ posição relativa (sentido medula-casca), em \%.

$\mathrm{O}$ melhor modelo foi selecionado com base nas estatísticas de $\mathrm{R}_{\text {aj. }}^{2}$ (Coeficiente de determinação), $\mathrm{S}_{\mathrm{yx}}$ (Erro padrão da estimativa), $\mathrm{F}_{\text {calc. }}$ (Valor de $\mathrm{F}$ calculado para o modelo) e análise gráfica dos resíduos. 
Em seguida, para cada modelo escolhido para descrever a retratibilidade radial nas árvores dominantes e médias, foram adicionadas variáveis Dummy, definidas pelas intensidades de desbaste, a fim de verificar a influência dos tratamentos aplicados.

$$
\mathrm{Ct}_{\mathrm{Rd}}, \log \left(\mathrm{Ct}_{\mathrm{Rd}}\right)=\mathrm{f}(\mathrm{P}, \mathrm{D}, \mathrm{D} . \mathrm{P})
$$

Em que: $\mathrm{Ct}_{\mathrm{Rd}}=$ retratibilidade radial das árvores médias e dominantes, em \%; $\mathrm{P}=$ posição relativa (sentido medula-casca) selecionada pelo procedimento Stepwise de regressão, em \%; D = tratamentos (dummy); D.P = interação tratamento (dummy) com a variável $P$.

Os dados médios de massa específica básica e massa seca das árvores dominantes e médias, em função da intensidade de desbaste, foram submetidos à análise de variância para delineamento experimental em blocos ao acaso, e, no caso de rejeição da hipótese de igualdade de médias entre tratamentos, ao teste de médias (Least Significant Difference - LSD, $\alpha=5 \%$ ).

\section{RESULTADOS E DISCUSSÃO}

\section{Parâmetros dendrométricos das árvores dominantes}

A intervenção silvicultural de desbaste em diferentes intensidades não influenciou as variáveis dendrométricas analisadas. Este resultado biometricamente já era esperado e confirma a Teoria de Assmann (1961) de que os desbastes, quando executados por baixo, não afetam o desenvolvimento das árvores dominantes, como pode ser observado na Tabela 2.

TABELA 2: Médias das características dendrométricas das árvores dominantes de Eucalyptus grandis.

TABLE 2: Dendrometric variables means of dominant trees of Eucalyptus grandis.

\begin{tabular}{c|c|c|c|c}
\hline \multirow{2}{*}{ Variável } & \multicolumn{4}{|c}{ Tratamentos } \\
\cline { 2 - 5 } & 1 & 2 & 4 & 8 \\
\hline DAP & $36,5 \mathrm{a}^{1}$ & $37,1 \mathrm{a}$ & $36,8 \mathrm{a}$ & $38,1 \mathrm{a}$ \\
$\mathrm{h}$ & $38,2 \mathrm{a}$ & $38,7 \mathrm{a}$ & $38,4 \mathrm{a}$ & $39,4 \mathrm{a}$ \\
$\mathrm{v}$ & $1,7610 \mathrm{a}$ & $1,8598 \mathrm{a}$ & $1,7935 \mathrm{a}$ & $1,9984 \mathrm{a}$ \\
\hline
\end{tabular}

Em que: $\mathrm{DAP}=$ diâmetro à altura do peito com casca, em $\mathrm{cm} ; \mathrm{h}=$ altura total, em $\mathrm{m} ; \mathrm{v}=$ volume total com casca, em $\mathrm{m}^{3}$. ${ }^{1}$ Médias seguidas por letras iguais em cada linha não diferem significativamente entre si em nível de $5 \%$ de probabilidade de erro.

\section{Parâmetros dendrométricos das árvores médias}

Os resultados dos parâmetros dendrométricos das árvores médias (Tabela 3) indicam uma tendência significativa de aumento do diâmetro médio com o aumento do espaço vital, confirmando a maioria dos resultados encontrados na literatura, como Monteiro et al. (1984); Blanco et al. (1988) e Berger (2000).

TABELA 3: Médias das características dendrométricas das árvores médias de Eucalyptus grandis.

TABLE 3: Dendrometric variables means of average trees of Eucalyptus grandis.

\begin{tabular}{c|c|c|c|c}
\hline \multirow{2}{*}{ Variável } & \multicolumn{4}{|c}{ Tratamentos } \\
\cline { 2 - 5 } & 1 & 2 & 4 & 8 \\
\hline DAP & $21,1 \mathrm{c}^{1}$ & $23,9 \mathrm{c}$ & $28,3 \mathrm{~b}$ & $34,5 \mathrm{a}$ \\
$\mathrm{h}$ & $28,1 \mathrm{c}$ & $29,7 \mathrm{c}$ & $32,4 \mathrm{~b}$ & $36,7 \mathrm{a}$ \\
$\mathrm{V}$ & $0,4399 \mathrm{c}$ & $0,5847 \mathrm{c}$ & $0,8947 \mathrm{~b}$ & $1,5175 \mathrm{a}$ \\
\hline
\end{tabular}

Em que: DAP = diâmetro à altura do peito com casca, em $\mathrm{cm} ; \mathrm{h}=$ altura total, em $\mathrm{m} ; \mathrm{v}=$ volume total com casca, em $\mathrm{m}^{3}$. ${ }^{1}$ Médias seguidas por letras iguais em cada linha não diferem significativamente entre si em nível de $5 \%$ de probabilidade de erro.

O acréscimo em diâmetro médio obtido entre o tratamento 1, testemunha sem desbaste e o tratamento 8 (média de 192 árvores/ha) foi de $38,8 \%$, ou seja, ocorreu um incremento de $13,4 \mathrm{~cm}$. Nos tratamentos 4 (média de 589 árvores/ha) e 2 (média de 1150 árvores/ha), quando comparados com a testemunha sem desbaste, também pode ser observado esse acréscimo em diâmetro, com valores de $25,4 \%(7,2 \mathrm{~cm})$ e $11,3 \%$ $(2,8 \mathrm{~cm})$, respectivamente.

Comparando-se as médias para altura total, pode-se verificar a tendência positiva desta variável com 
o aumento da intensidade de desbaste (Tabela 3). Resultados semelhantes foram encontrados por Lopez e Aparício (1995), que relacionaram as diferenças de altura encontradas com o nível de competição entre os indivíduos, concluindo que o maior número de árvores dominadas resulta em uma tendência de alturas médias menores nos povoamentos mais densos.

Com relação ao volume total com casca, os resultados apresentados são semelhantes aos encontrados por Berger (2000) e Fishwick (1976), os quais observaram que os maiores espaços vitais ocasionaram maiores volumes individuais (Tabela 3). O desenvolvimento em diâmetro e altura nos maiores espaços vitais também contribuiu significativamente para a obtenção de maiores volumes individuais nesses espaçamentos.

\section{Modelagem do volume total com casca}

Para a análise do volume total com casca por árvore, em função das variáveis consideradas de fácil obtenção (DAP e h), foram agrupadas as árvores dominantes e médias de todos os tratamentos. Para descrever o volume, foi utilizado o modelo de Spurr, que apresentou valor de $\mathrm{F}_{\mathrm{cal}}=1262,53$, significativo em nível de probabilidade. $>\mathrm{F}=0,0001$, um excelente ajuste, com um coeficiente de determinação ajustado igual a 0,98 e erro padrão da estimava igual a 0,0947 . O modelo resultante para estimar o volume com casca das árvores é expresso por:

$$
\mathrm{v}=0,01208170+0,00003407 \mathrm{~d}^{2} \mathrm{~h}
$$

Aos dados de volume com casca ajustados em função da variável independente $d^{2} h$, foram adicionadas a variável Dummy, definida pelos tratamentos de intensidade de desbaste. O resultado da análise de variância indicou não haver diferença significativa a 5\% de probabilidade de erro para níveis e inclinação das tendências dos volumes com casca estimados em função das variáveis Dummy. Portanto, as estimativas de volume com casca podem ser realizadas unicamente com a variável independente $\mathrm{d}^{2} h$.

Esta equação também foi selecionada por Paula Neto (1975), a qual, estudando 127 modelos volumétricos lineares, definiu o modelo de Spurr para estimar o volume de Eucalyptus saligna. A equação permite estimar o volume com casca a partir do diâmetro à altura do peito e altura correspondente, considerando, portanto, a variação da relação $\mathrm{h} / \mathrm{d}$ e, com isso, a forma do tronco das árvores ou do próprio fator de forma.

\section{Parâmetros tecnológicos das árvores dominantes}

\section{Massa específica básica ponderada}

Os dados de massa específica básica ponderada em função do volume ( $\rho_{\mathrm{b}}$ pond.) das árvores dominantes, submetidos à análise de variância, não foram influenciados pelas diferentes intensidades de desbaste, com valor de $\mathrm{F}_{\mathrm{cal}}=0,60$ e nível de probabilidade de erro não-significativo (Prob. $>\mathrm{F}=0,632$ ).

O resultado médio deste parâmetro tecnológico, para todos os tratamentos, aos 14 anos de idade, foi de $0,413 \mathrm{~g} / \mathrm{cm}^{3}$. Busnardo et al. (1987) e Ferreira (1996) encontraram resultados semelhantes, com valores de $\rho_{\mathrm{b} \text { pond. }}=0,410 \mathrm{~g} / \mathrm{cm}^{3}$ e $0,430 \mathrm{~g} / \mathrm{cm}^{3}$, respectivamente, porém, para madeira de Eucalyptus grandis com 8 anos de idade.

Já Lopes (2003), analisando 63 árvores matrizes desta mesma espécie, encontrou uma média para este parâmetro tecnológico de $0,460 \mathrm{~g} / \mathrm{cm}^{3}$, aos 26 anos de idade. Essa diferença, segundo Kollmann e Côté (1968) e Panshin e De Zeeuw (1980), pode ser atribuída a fatores ambientais ou genéticos.

\section{Massa seca}

O parâmetro tecnológico massa seca (MS) das árvores dominantes, submetido à análise de variância, não mostrou diferenças entre os tratamentos. $\mathrm{O}$ valor de $\mathrm{F}_{\text {calc. }}=0,20$ e o nível de probabilidade de erro nãosignificativo (Prob. $>\mathrm{F}=0,890$ ) para o fator intensidade de desbaste demonstraram a inexistência de influência significativa sobre a massa seca das árvores, aos 14 anos de idade.

Este resultado já era esperado, visto que o volume e a massa específica básica das árvores dominantes também não foram influenciados pelos tratamentos empregados. 


\section{Retratibilidade}

A propriedade física de contração radial total em função da posição relativa no sentido medula-casca das árvores dominantes, modelada com o procedimento Stepwise de regressão, apresentou $F_{\text {cal }}=57,07$, significativo em nível de probabilidade $>\mathrm{F}=0,0001$, com um coeficiente de determinação ajustado igual a 0,41 e erro padrão da estimava igual a 0,205 . O modelo resultante para estimar a contração radial total das árvores é expresso por:

$$
\mathrm{Ct}_{\mathrm{Rd}}=\mathrm{e}^{1,14135+0,00690 \mathrm{P}}
$$

Os dados de contração radial total das árvores dominantes, submetidos à análise de regressão com o emprego de variável Dummy, evidenciaram que o modelo selecionado pode ser utilizado para todas as intensidades de desbaste, pois, nenhuma das Dummy empregadas para verificar a influência dos tratamentos na contração radial total foram significativas em nível $\alpha=5 \%$ de probabilidade de erro.

Após a escolha do modelo e a constatação de que a intensidade de desbaste não influencia a contração radial total das árvores dominantes, foi verificada a variação desta característica no sentido medula-casca. A Figura 1 mostra que a retratibilidade da madeira de Eucalyptus grandis, aos 14 anos de idade, na altura do DAP, apresenta menores valores na região da medula $(3,5 \%)$ com acréscimo até a região mais periférica do tronco $(5,6 \%)$.

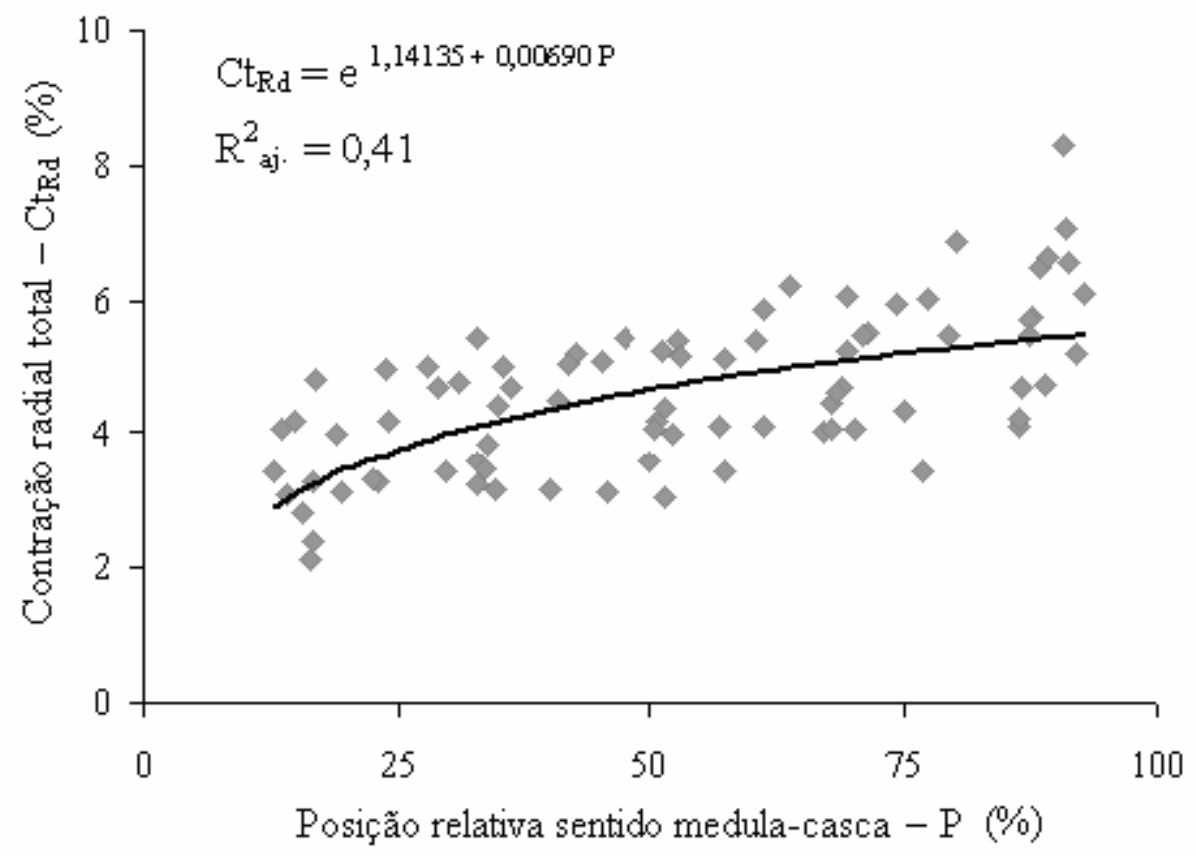

FIGURA 1: Variação da contração radial total no sentido medula-casca das árvores dominantes.

FIGURE 1: Variation of the radial shrinkage from pith-to-bark direction of the dominant trees.

Os valores inferiores dos coeficientes de contração volumétrica e linear na região medular, segundo Oliveira e Silva (2003), podem ocorrer devido à existência de grandes ângulos microfibrilares, que são comuns nas regiões mais internas do tronco, acarretando, conseqüentemente, maiores contrações longitudinais e menores coeficientes de contrações na direção transversal.

\section{Parâmetros tecnológicos das árvores médias}

\section{Massa específica básica ponderada}

No resultado dos dados de massa específica básica ponderada em função do volume $\left(\rho_{\mathrm{b} \text { pond. }}\right)$ das árvores médias, descritos na Tabela 4, observa-se que não existe interação (positiva ou negativa) significativa

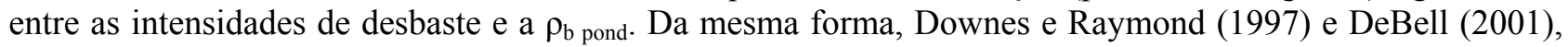
citando vários autores, evidenciam resultados bastante contraditórios de uma possível interação entre esta característica tecnológica e os tratamentos silviculturais. 
TABELA 4: Comparação de médias do parâmetro massa específica básica das árvores médias em função da intensidade de desbaste.

TABLE 4: Comparison of average of the parameter basic density of the average trees in function of the thinning intensity.

\begin{tabular}{c|c|c}
\hline Tratamentos & $\rho_{\mathrm{b} \text { pond. }}$ & LSD \\
\hline 4 & 0,434 & $\mathrm{a}^{1}$ \\
1 & 0,411 & $\mathrm{ab}$ \\
8 & 0,393 & $\mathrm{~b}$ \\
2 & 0,392 & $\mathrm{~b}$ \\
\hline
\end{tabular}

Em que: 1 = Médias seguidas por letras iguais na coluna não diferem significativamente entre si; $\rho_{\mathrm{b} \text { pond }}=$ média da massa específica básica ponderada das árvores médias, em g/ $\mathrm{cm}^{3} ; \mathrm{LSD}=$ Least Significant Difference.

Várias investigações silviculturais têm mostrado que tratamentos que aceleram o nível de crescimento também tendem a aumentar a massa específica básica. É o que evidenciam Wilkins e Kitahara (1991) e Malan e Hoon (1992), em seus resultados com Eucalyptus grandis. Contudo, Downes e Raymond (1997) relatam que esta relação não é perfeita, ou melhor, os tratamentos que aumentam o volume de produção nem sempre aumentam esta característica tecnológica.

\section{Massa seca}

Os resultados dos dados de massa seca (MS) das árvores médias, submetidos à análise de variância, aos 14 anos de idade, mostram que este parâmetro tecnológico foi influenciado significativamente pela intensidade de desbaste em nível $\alpha=5 \%$ de probabilidade de erro e valor de $\mathrm{F}_{\text {calc. }}=43,96$. $\mathrm{O}$ teste de comparação de médias para o fator intensidade de desbaste, com as médias de massa seca, é demonstrado na Tabela 5.

TABELA 5: Comparação de médias do parâmetro massa seca das árvores médias em função da intensidade de desbaste.

TABLE 5: Comparison of average of the parameter dry mass of the average trees in function of the thinning intensity.

\begin{tabular}{c|c|c}
\hline Tratamentos & MS & LSD \\
\hline 8 & 449,7 & $\mathrm{a}^{1}$ \\
4 & 291,3 & $\mathrm{~b}$ \\
2 & 114,0 & $\mathrm{c}$ \\
1 & 92,2 & $\mathrm{c}$ \\
\hline
\end{tabular}

Em que: 1 = Médias seguidas por letras iguais na coluna não diferem significativamente entre si; $\mathrm{MS}=$ média da massa seca das árvores médias, em kg; LSD = Least Significant Difference.

Ao comparar as médias de MS, percebe-se que, quanto maior a intensidade dessa intervenção silvicultural, maior é esse parâmetro tecnológico. Com o aumento do espaço vital, ocorre uma tendência significativa de aumento da massa seca das árvores médias.

\section{Retratibilidade}

A contração radial total das árvores médias em função da posição relativa no sentido medula-casca, modelada com procedimento Stepwise de regressão, apresentou $F_{\text {cal }}=68,04$, significativo em nível de probabilidade. $>\mathrm{F}=0,0001$, com um coeficiente de determinação ajustado igual a 0,54 e erro padrão da estimava igual a 0,190 . O modelo resultante para estimar a contração radial total das árvores é expresso por:

$$
\mathrm{Ct}_{\mathrm{Rd}}=\mathrm{e}^{2,56638-4,09945 / \log \mathrm{P}}
$$

Os dados de contração radial total das árvores médias, submetidos à análise de regressão com o emprego de variável Dummy, também evidenciaram que o modelo selecionado pode ser utilizado para todas as intensidades de desbaste, pois nenhuma das Dummy empregadas para verificar a influência dos tratamentos na contração radial total foram significativas em nível $\alpha=5 \%$ de probabilidade de erro. 
Após a escolha do modelo e a constatação de que a intensidade de desbaste não influencia a contração radial total das árvores médias, foi verificada a variação desta característica no sentido medulacasca. A Figura 2 mostra que a retratibilidade da madeira das árvores médias de Eucalyptus grandis, aos 14 anos de idade, na altura do DAP, também apresenta menores valores na região da medula $(2,6 \%)$, com acréscimo até a região mais periférica do tronco $(5,2 \%)$.

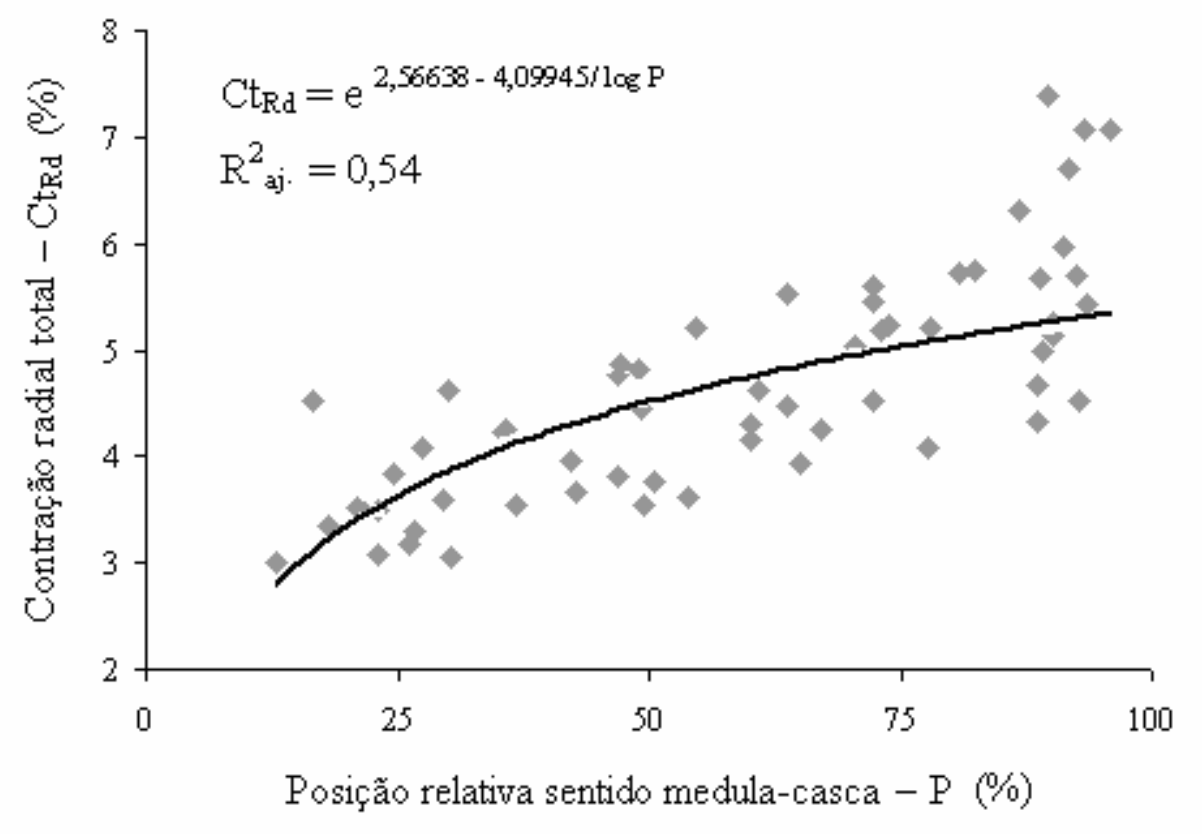

FIGURA 2: Variação da contração total radial no sentido medula-casca das árvores médias.

FIGURE 2: Radial shrinkage from pith-to-bark direction of the average trees.

\section{CONCLUSÕES}

Os resultados obtidos neste estudo, para Eucalyptus grandis, aos 14 anos, permitem concluir que:

Os desbastes quando aplicados por baixo não influenciam as dimensões das árvores dominantes, porém alteram significativamente as dimensões das árvores médias.

A massa específica ponderada das árvores dominantes não é influenciada pelo desbaste, sendo a madeira classificada como leve a moderadamente leve. Para as árvores médias, contudo, varia significativamente com a aplicação de desbastes, sem apresentar tendência com a intensidade dessa intervenção silvicultural.

O emprego de desbaste pesado aumenta a produção de massa seca das árvores médias, não influenciando as árvores dominantes.

A contração radial total da madeira na altura do DAP das árvores dominantes e médias não é influenciada pela intensidade de desbaste; porém, varia no sentido medula-casca, apresentando menores valores na região da medula com acréscimo até a região mais periférica do tronco.

\section{AGRADECIMENTOS}

Os autores agradecem o apoio da Empresa Flosul Indústria e Comércio de Madeiras Ltda., em especial, ao Setor Florestal, pela colaboração para a realização deste trabalho.

\section{REFERÊNCIAS BIBLIOGRÁFICAS}

ASSMANN, E. Waldertragskunde. Muenchen: BLV Verlagsgessellschaft, 1961. 435p.

BERGER, R. Crescimento e qualidade da madeira de um clone de Eucalyptus saligna Smith sob o efeito do espaçamento e da fertilização. 2000. 126f. Dissertação (Mestrado em Engenharia Florestal) - Universidade Federal de Santa Maria, Santa Maria, 2000. 
BLANCO, J.J.; RAMOS, L., ROMERO, F.A. et al. Comportamiento de Pinus tropicalis en cinco espaciamentos de plantación en los suelos ferraliticos cuarciticos amarillos de Viñales, Cuba. Revista Forestal Baracoa, v.18, n.2, p.21$30,1988$.

BUSNARDO, C.A.; GONZAGA, J.V.; FOELKEL, C.E.B. et al. Em busca da qualidade ideal da madeira do eucalipto para produção de celulose, IV. Altura ideal de amostragem para avaliação da densidade média para árvores de Eucalyptus grandis. Guaíba: Riocell S.A., 1987. 18p. (Relatório Técnico)

DEBELL, D.S.; KEYES, C.R.; GARTNER, B.L. Wood density of Eucalyptus saligna grown in Hawaiian plantations: effects of silvicultural practices and relation to growth rate. Australian Forestry, v.64, n.2, p.106-110, 2001.

DOWNES, G.M.; RAYMOND, C.A. Variation in wood density in plantation eucalypts. Sampling plantation eucalypts for wood and fibre properties. Australia: 1997. p. 88-99.

FERREIRA, G.W. Qualidade da celulose kraft-antraquinona de Eucalyptus dunnii Maiden plantado em cinco espaçamentos em relação ao Eucalyptus grandis Hill ex Maiden e Eucalyptus saligna Smith. $1996.135 f$. Dissertação (Mestrado em Engenharia Florestal) - Universidade Federal de Santa Maria, Santa Maria, 1996.

FINGER, C.A.G. Fundamentos de biometria florestal. Santa Maria: UFSM/ CEPEF/ FATEC, 1992. 269 p.

FISHWICK, R.W. Estudo de espaçamentos e desbastes em plantações brasileiras. Brasil Florestal, Brasília, v.7, p. 1323, 1976.

HASELEIN, C.R.; BERGER, R.; GOULART, M. et al. Propriedades de flexão estática da madeira úmida e a $12 \%$ de umidade de um clone de Eucalyptus saligna Smith quando submetido a diferentes espaçamentos e doses de adubação. Ciência Florestal, Santa Maria, v. 12, n. 2, p. 147-152, 2002.

KOLLMANN, F.F.P.; CÔTÉ, W.A. Principles of wood science and technology. Berlin: Springer, 1968. v. 1. 592p.

LOPES, M.C. Agrupamento de árvores matrizes de Eucalyptus grandis em função das variáveis dendrométricas e das características tecnológicas da madeira. 2003. 93f. Dissertação (Mestrado em Engenharia Florestal) Universidade Federal de Santa Maria, Santa Maria, 2003.

LOPEZ, J.A.; APARICIO, J.L. Crecimiento y producción de Eucalyptus grandis com diferentes densidades de plantación (Información hasta el 120 año de edad). Bella Vista : INTA, 1995. 12p.

MALAN, F.S.; HOON, M. Effect of initial spacing and thinning on some wood properties of Eucalyptus grandis. South African Forestry Journal, n.163, p.13-20, 1992.

MONTEIRO, R.F.R.; CORDEIRO, J.A.; KIKUTI, P. Competição entre espaçamentos com Eucalyptus dunnii Maiden. In: CONGRESSO FLORESTAL ESTADUAL, 5., 1984, Nova Prata. Anais (CD) ... Nova Prata: Prefeitura Municipal, 1984.

OLIVEIRA, J.T.S. Caracterização da madeira de eucalipto para a construção civil. 1998. 429p. Tese (Doutorado) - Escola Politécnica, Universidade de São Paulo, São Paulo, 1998.

OLIVEIRA, J.T.S.; SILVA, J.C.R. Variação radial da retratibilidade e densidade básica da madeira Eucalyptus saligna Smith. Revista Árvore, Viçosa, v.27, n.3, p.381-385, 2003.

PANSHIN, A.J.; DE ZEEUW, C. Text book of wood technology. 4.ed. New York: Mc Graw Hill, 1980. 722p.

PAULA NETO, F.de. Construction of standart volume table for Eucalyptus saligna in the Iron Region of Brazil. 1975. 101 f. Tese (Ph.D.) - Purdue University, Lafayette, 1975.

SAS. Statistical Analysis System. Programa de computador, ambiente VM. Cary, 1993. Versão 6.08.

SCHEEREN, L.W. Estruturação da produção de povoamentos monoclonais de Eucalyptus saligna Smith manejados em alto fuste. 2003. 181f. Tese (Doutorado em Engenharia Florestal) - Universidade Federal de Santa Maria, Santa Maria, 2003.

SCHNEIDER, P.R. Manejo florestal : planejamento da produção florestal. Santa Maria: UFSM, 2002. 492p.

SCHNEIDER, P.R.; FINGER, C.A.G.; HOPPE, J.M. et al. Produção de Eucalyptus grandis Hill ex Maiden em diferentes intensidades de desbaste. Ciência Florestal, Santa Maria, v.8, n.1, p.129-140, 1998.

SILVA, J.C.; OLIVEIRA, J.T.S. Avaliação das propriedades higroscópicas da madeira de Eucalyptus saligna Smith. em diferentes condições de umidade relativa do ar. Revista Árvore, Viçosa, v.27, n.2, p. 233-239, 2003.

SMITH, D.M. The practice of silviculture. New York : John Wiley \& Sons, 1962. 578p.

SOARES, T.S.; SILVA M.L.; GAMA J.R.V. et al. Avaliação econômica de plantações de Eucalipto submetidas a desbaste. Revista Árvore, Viçosa, v.27, n.4, p.481-486, 2003. 
VITAL, B.R. Métodos de determinação da densidade da madeira. Viçosa: SIF/UFV, 1984. 21 p.

WILKINS, A.P.; KITAHARA, R. Silvicultural treatments and associated growth rates, growth strains and wood properties in 12,5-year-old Eucalyptus grandis. Australian Forestry, v.54, n.1/2, p. 99-104, 1991. 